PROCEEDINGS OF THE

AMERICAN MATHEMATICAL SOCIETY

Volume 125, Number 10, October 1997, Pages 3059-3067

S 0002-9939(97)04101-4

\title{
VOLUMES OF HYPERBOLIC HAKEN MANIFOLDS, II
}

\author{
MARC CULLER AND PETER B. SHALEN
}

(Communicated by James West)

\begin{abstract}
We show that if $M$ is a closed hyperbolic 3-manifold and if $\pi_{1}(M)$ has a non-abelian free quotient, then the volume of $M$ is greater than 0.92 . If, in addition, $\pi_{1}(M)$ contains no genus- 2 surface groups, then the volume of $M$ is greater than 1.02. Using these results we show that if there are infinitely many primitive homology classes in $H_{2}(M ; \mathbb{Z})$ which are not represented by fibroids, then the volume of $M$ is greater than 0.83 .
\end{abstract}

\section{INTRODUCTION}

In the paper [CHS] it is shown that if $M$ is a closed hyperbolic 3-manifold of minimal volume, then the first Betti number of $M$ is at most 2. A lower bound of 0.34 for the volume of a manifold with first Betti number 2 was given in [CS2]. This bound is considerably smaller than the smallest known volume of a closed hyperbolic 3-manifold, which is about 0.94 and is realized by an example discovered by Weeks $[\mathrm{W}]$. In this paper we give stronger lower bounds which apply to certain classes of hyperbolic 3-manifolds, including many manifolds with first Betti number 2 . These bounds are close to the volume of Weeks's example, and some of them exceed it.

Our first lower bound, given by Theorem 1.3, applies to any closed hyperbolic 3manifold $M$ whose fundamental group has a non-abelian free quotient. (Such a free quotient exists if and only if $M$ contains two disjoint closed surfaces that represent independent classes in $H_{2}(M ; \mathbb{Q})$.) Theorem 1.3 asserts that if $\pi_{1}(M)$ has a nonabelian free quotient, then $M$ contains a ball of radius $(\log 3) / 2$. Estimates for the density of a sphere-packing in hyperbolic space then imply that the volume of $M$ is greater than 0.92. We also point out, in Theorem 1.4, that the conclusion of Theorem 1.3 can be strengthened under the additional hypothesis that $\pi_{1}(M)$ has no subgroup isomorphic to the fundamental group of a genus-2 surface. Specifically, if $\pi_{1}(M)$ has a non-abelian free quotient and contains no genus-2 surface groups, then the volume of $M$ is greater than 1.02, and hence exceeds the volume of Weeks's example. An even stronger estimate applies if the free quotient has rank $>2$.

Our second main result applies to many manifolds of Betti number 2. To indicate the statement, we recall some definitions from [CS3]. A compact, connected, orientable topological 3-manifold (with boundary) $N$ is called a book of I-bundles if it has the form $N=E \cup V$, where

(i) $E$ is an $I$-bundle over a non-empty compact 2-manifold-with-boundary $B$;

Received by the editors November 6, 1995.

1991 Mathematics Subject Classification. Primary 57M50; Secondary 57N10.

This research was partially supported by National Science Foundation grant DMS9302520.

(C)1997 American Mathematical Society 
(ii) each component of $V$ is homeomorphic to $D^{2} \times S^{1}$;

(iii) the set $A=E \cap V$ is the inverse image of $\partial B$ under the bundle projection $E \rightarrow B ;$ and

(iv) each component of $A$ is an annulus in $\partial V$ which is homotopically non-trivial in $V$.

A fibroid in a closed orientable 3-manifold $M$ is a closed incompressible surface such that each component of the manifold obtained by splitting $M$ along $S$ is a book of I-bundles. (This definition is slightly more general than the one used in [CS3]. A "fibroid" in the sense of [CS3] is a fibroid in the above sense which is connected.) A corollary to one of the main results of this paper, Corollary 3.4, asserts that if $M$ is a closed, orientable hyperbolic 3-manifold whose first Betti number $\beta_{1}(M)$ is at least 2 and if $M$ contains no non-separating, connected fibroid, then the volume of $M$ is greater than 0.83 . The hypothesis that $M$ contains no non-separating, connected fibroid is to be regarded as a slightly stronger version of the condition that $M$ not be a fibered 3-manifold.

The theorem from which Corollary 3.4 is deduced, Theorem 3.3, asserts that the same volume estimate holds under more general conditions. An element of an abelian group $A$ will be termed primitive if it cannot be written in the form $n x$ with $x \in A$ and $n>1$. Theorem 3.3 asserts that if there are infinitely many primitive homology classes in $H_{2}(M ; \mathbb{Z})$ which are not represented by connected fibroids, then the volume of $M$ is greater than 0.83 .

Theorem 3.3 and Corollary 3.4 are interesting only when $\beta_{1}(M)=2$. If $\beta_{1}(M)<$ 2 , then $H_{2}(M ; \mathbb{Z})$ contains at most two primitive elements, and if $\beta_{1}(M)>2$, then the results of [CS1] and [CHS] give larger lower bounds for the volume of $M$.

The hypothesis of Theorem 3.3 is related to the norm on $H_{2}(M ; \mathbb{R})$ constructed by Thurston in $[\mathrm{T}]$. We regard $H_{2}(M ; \mathbb{Z})$ as the integer lattice in $H_{2}(M ; \mathbb{R})$. In the case that $M$ is hyperbolic, Thurston's construction gives a norm on $H_{2}(M ; \mathbb{R})$ whose unit sphere is a finite-sided polyhedron. He shows that there is a finite collection of top-dimensional faces of this polyhedron, called fibered faces, such that a ray from the origin contains a primitive class in $H_{2}(M ; \mathbb{Z})$ which is represented by a fiber if and only if it meets the unit sphere in the interior of a fibered face. In the case that $\beta_{1}(M)=2$ the primitive classes (up to sign) in $H_{2}(M ; \mathbb{Z}$ ) are identified with the points of $P^{1}(\mathbb{Q}) \subset P^{1}(\mathbb{R})$, and it follows from Thurston's theorem that the classes that are represented by fibers form the intersection of $P^{1}(\mathbb{Q})$ with a finite union of open intervals. L. Mosher has recently proved a partial analogue of this result: he has shown that those primitive classes in $H_{2}(M ; \mathbb{Z}$ ) (up to sign) which are represented by fibroids form an open subset of $P^{1}(\mathbb{Q}) \subset P^{1}(\mathbb{R})$ in the subspace topology. If this set has an infinite complement, then there are infinitely many primitive classes in $H_{2}(M ; \mathbb{Z})$ which are not represented by fibroids. In particular, there are infinitely many primitive classes which are not represented by connected fibroids, so that Theorem 3.3 gives a lower bound of 0.83 for the volume of $M$.

The proof of Theorem 3.3 is divided into two cases, according to whether $\pi_{1}(M)$ has a non-abelian free quotient. In the case where it has a non-abelian free quotient, the conclusion follows immediately from Theorem 1.3. In the case where $\pi_{1}(M)$ has no non-abelian free quotient, it follows from the elementary arguments given in Section 2 that every primitive element of $H_{2}(M ; \mathbb{Z})$ is represented by some connected incompressible surface. In this case, the conclusion follows from a result, Theorem 3.1, which, in this somewhat more restricted setting, is stronger than 
Theorem 3.3: it asserts that if there are infinitely many primitive homology classes that can be represented by connected incompressible surfaces that are not fibroids, then the volume of $M$ is greater than 0.83 .

The proofs in this paper are conceptual and qualitative. They are based on more technical results that were proved in [CS1], [CS2], [CS3], [ACCS] and [CHS], and we hope that they illustrate the power of the techniques developed in those papers.

Hyperbolic distance in $\mathbb{H}^{3}$ will be denoted dist. A hyperbolic ball in a hyperbolic 3-manifold is defined to be a connected open subset which, with its intrinsic metric, is isometric to a ball in $\mathbb{H}^{3}$.

We thank Lee Mosher for explaining his work on the set of homology classes represented by fibroids, which we discussed above.

\section{Section 1. Free quotients and hyperbolic volume}

The main result of this section is Theorem 1.3, which applies to a manifold whose fundamental group has a non-abelian free quotient. We also establish a result, Theorem 1.4, that gives a stronger estimate under the additional assumption that the fundamental group contains no genus- 2 surface groups. The statements of these results are related but the proofs are quite different.

The following two group-theoretical results are needed for the proof of Theorem 1.3. We will use the notation $|S|$ to denote the cardinality of a set $S$.

Proposition 1.1. Let $\Gamma$ be a group which admits a homomorphism onto a noncyclic finite group $G$, and let $S$ be a collection of elements of $\Gamma$. Suppose that every pair of distinct elements of $S$ generate $\Gamma$. Then $|S| \leq|G|-1$.

Proof. Since $G$ is non-cyclic we certainly have $|G|>1$. Thus the assertion is true if $|S| \leq 1$. Now assume that $|S|>1$, and let $h$ denote a homomorphism of $\Gamma$ onto $G$. For any two distinct elements $s$ and $s^{\prime}$ of $S$, the hypothesis implies that $s$ and $s^{\prime}$ generate $\Gamma$, and hence that $h(s)$ and $h\left(s^{\prime}\right)$ generate $G$. Since $G$ is non-cyclic it follows that $h(s) \neq h\left(s^{\prime}\right)$ and that $h(s) \neq 1$. Thus we have shown that $h \mid S$ is one-to-one and that $h(S) \subset G-\{1\}$. Thus $|S| \leq|G|-1$.

Proposition 1.2. Let $\Gamma$ be a group which has a non-abelian free quotient. Let $S$ be a subset of $\Gamma$ with $|S|=4$. Then there exist a subgroup $K$ of finite index in $\Gamma$ and an epimorphism $\phi: K \rightarrow \mathbb{Z}$ such that at least two elements of $S$ are contained in the kernel of $\phi$.

Proof. Let $h$ be a homomorphism of $\Gamma$ onto a free group $\bar{\Gamma}$ of rank $n \geq 2$. We claim that there exist two distinct elements $s$ and $s^{\prime}$ of $S$ such that $h(s)$ and $h\left(s^{\prime}\right)$ generate a proper subgroup of $\bar{\Gamma}$. This is clear if the restriction of $h$ to $S$ fails to be one-toone. If $\left.h\right|_{S}$ is one-to-one, then $|h(S)|=4$ and, since $\bar{\Gamma}$ admits a homomorphism onto the non-cyclic group $G$ of order 4 , it follows from Proposition 1.1 that there exist two distinct elements of $h(S)$ which generate a proper subgroup $F$ of $\Gamma$. (Otherwise Proposition 1.1 would imply that $|S|=|h(S)| \leq 3$.)

We now apply M. Hall's theorem (see $[\mathrm{H}],[\mathrm{KS}]$ ) that every finitely generated subgroup of a finite-rank free group $\bar{\Gamma}$ is a free factor of some finite-index subgroup of $\bar{\Gamma}$. Thus there is a subgroup $\bar{K}$ of some index $d<\infty$ in $\bar{\Gamma}$ such that $F \leq \bar{K}$ and $F$ is a free factor of $\bar{K}$.

We claim that $F$ is a proper free factor of $\bar{K}$. Suppose to the contrary that $F=\bar{K}$. Then $F$ also has index $d$ in $\bar{\Gamma}$. Since $F$ is a proper subgroup of $\Gamma$ we must have $d>1$. On the other hand the rank of $F$ is $(n-1) d+1$ and, since $F$ is 
generated by two elements, we must have $(n-1) d+1 \leq 2$. But this is impossible since $n \geq 2$.

Since $F$ is a proper free factor of $\bar{K}$ there is an epimorphism $\phi: \bar{K} \rightarrow \mathbb{Z}$ whose kernel contains $F$. If we set $K=h^{-1}(\bar{K})$, then $K$ has finite index in $\Gamma$ and $\phi \circ h$ is an epimorphism from $K$ to $\mathbb{Z}$ whose kernel contains $h^{-1}(F)$, and hence contains two distinct elements of $S$.

Theorem 1.3. Let $M$ be a closed, orientable hyperbolic 3-manifold such that $\pi_{1}(M)$ has a non-abelian free quotient. Then $M$ contains a hyperbolic ball of radius $(\log 3) / 2$. Hence the volume of $M$ exceeds 0.92 .

Proof. According to [CHS, Proposition 2.5], if $\Gamma$ is a co-compact, torsion-free, discrete subgroup of $\operatorname{Isom}_{+}\left(\mathbb{H}^{3}\right)$, and $\Delta$ is a positive real number, then either (i) the closed hyperbolic 3-manifold $M=\mathbb{H}^{3} / \Gamma$ contains a hyperbolic ball of radius $\Delta / 2$, or (ii) there exist a point $z_{0} \in \mathbb{H}^{3}$ and pairwise non-commuting (and hence distinct) elements $\xi_{0}, \xi_{1}, \xi_{2}, \xi_{3}$ of $\Gamma$ such that $\operatorname{dist}_{h}\left(z_{0}, \xi_{i} \cdot z_{0}\right)<\Delta$ for $i=0,1,2,3$.

Now suppose that $M$ is a closed hyperbolic 3-manifold such that $\pi_{1}(M)$ has a non-abelian free quotient, but that $M$ contains no hyperbolic ball of radius $(\log 3) / 2$. Let us write $M=\mathbb{H}^{3} / \Gamma$, where $\Gamma \leq \operatorname{Isom}_{+}\left(\mathbb{H}^{3}\right)$ is co-compact, discrete and torsionfree. Then we may fix a point $z_{0} \in \mathbb{H}^{3}$ such that (ii) holds with $\Delta=\log 3$.

Since $\Gamma \cong \pi_{1}(M)$ has a non-abelian free quotient, we may apply Proposition 1.2 to the family $\left(\xi_{i}\right)_{i=1}^{4}$. This shows that, after re-indexing the $\xi_{i}$ if necessary, we may assume that there exist a subgroup $K$ of finite index in $\Gamma$ and an epimorphism $\phi: K \rightarrow \mathbb{Z}$ such that $\xi_{1}, \xi_{2} \in K$ and $\phi\left(\xi_{1}\right)=\phi\left(\xi_{2}\right)=0$.

We wish to apply Theorem 9.1 of [CS1] to $\xi_{1}$ and $\xi_{2}$. The latter theorem applies to two non-commuting elements of $\operatorname{Isom}_{+}\left(\mathbb{H}^{3}\right)$ which generate a discrete, torsionfree non-co-compact, purely loxodromic, topologically tame group. We know that $\xi_{1}$ and $\xi_{2}$ do not commute. Since $\xi_{1}$ and $\xi_{2}$ belong to $\Gamma$, they generate a discrete, torsion-free, purely loxodromic group $F$. The group $F$ has infinite index in $\Gamma$ because it is contained in the kernel of the epimorphism $\phi: K \rightarrow \mathbb{Z}$; hence $F$ is non-co-compact. Finally, by [CS2, Prop. 2.2] and [CS1, Prop. 10.2], if $\phi$ is any epimorphism from the fundamental group of a closed hyperbolic 3-manifold to $\mathbb{Z}$, then any two-generator subgroup of the kernel of $\phi$ is topologically tame. Thus $\xi_{1}$ and $\xi_{2}$ satisfy all the hypotheses of [CS1, Theorem 9.1]. The latter theorem now asserts that for any point $z \in \mathbb{H}^{3}$ we have

$$
\max \left\{\operatorname{dist}\left(z, \xi_{1} \cdot z\right), \operatorname{dist}\left(z, \xi_{2} \cdot z\right)\right\} \geq \log 3 .
$$

But for $z=z_{0}$ this contradicts the choice of the elements $\xi_{1}$ and $\xi_{2}$.

This completes the proof that, under the hypotheses of Theorem 1.3, the manifold $M$ contains a hyperbolic ball of radius $(\log 3) / 2$. The lower bound 0.92 for the volume of $M$ now follows from sphere-packing arguments as in [CS1, Corollary 10.4].

The following result gives stronger estimates than Theorem 1.3 under somewhat stronger hypotheses.

Theorem 1.4. Let $M$ be a closed orientable hyperbolic 3-manifold such that $\pi_{1}(M)$ has no subgroup isomorphic to the fundamental group of a closed, orientable surface of genus 2 .

(i) If $\pi_{1}(M)$ has a non-abelian free quotient, then the volume of $M$ exceeds 1.02 . 
(ii) If $\pi_{1}(M)$ admits a homomorphism onto a free group of rank 3 , then the volume of $M$ exceeds 1.54 .

(iii) If $\pi_{1}(M)$ admits a homomorphism onto a free group of rank 4 , then the volume of $M$ exceeds 3.08 .

Proof. Assertion (iii) is a special case of [ACCS, Corollary 9.2], which asserts that if the first Betti number of the closed, orientable, hyperbolic 3-manifold $M$ is at least 4 and if $\pi_{1}(M)$ has no subgroup isomorphic to the fundamental group of a closed, orientable surface of genus 2, then the volume of $M$ is greater than 3.08. To prove (i) we consider a homomorphism $h$ of $\pi_{1}(M)$ onto a free group $F$ of rank 2 , and we choose any index-3 subgroup $F_{0}$ of $F$. Then $\Gamma_{0}=h^{-1}\left(F_{0}\right)$ has index 3 in $\pi_{1}(M)$, and hence defines a 3 -sheeted covering space $M_{0}$ of $M$. The group $\pi_{1}\left(M_{0}\right) \cong \Gamma_{0}$ admits a homomorphism onto $F_{0}$, which is a free group of rank 4 . Of course $\pi_{1}\left(M_{0}\right)$ contains no genus-2 surface groups since $\pi_{1}(M)$ contains none. Hence by assertion (iii) we have $\operatorname{vol}\left(M_{0}\right)>3.08$, and hence

$$
\operatorname{vol}(M)=\frac{\operatorname{vol}\left(M_{0}\right)}{3}>1.02 .
$$

Assertion (ii) is deduced in the same way from assertion (iii), since an index-2 subgroup of a rank-3 free group has rank $5 \geq 4$.

\section{Section 2. Free quotients vs. COnNeCted surfaces}

In this section we establish the following elementary result, which will be used in the proof of Theorem 3.3.

Proposition 2.1. Let $M$ be a closed, orientable 3-manifold such that $\pi_{1}(M)$ has no non-abelian free quotient. Then every primitive element of $H_{2}(M ; \mathbb{Z})$ is represented by a connected, oriented, incompressible surface.

Proof. Let $c$ be any element of $H_{2}(M ; \mathbb{Z})$. According to [T, Lemma 1], $c$ can be represented by some oriented 2-manifold $S$. (To say that $S$ is oriented means that each of its components is oriented and hence represents an element of $H_{2}(M ; \mathbb{Z})$; the sum of these elements is equal to $c$.) After doing a finite number of surgeries on $S$, which do not change the homology class represented by $S$, we may assume that each component of $S$ is incompressible. We may also assume that no component of $S$ separates the manifold $M$, since discarding the separating components of $S$ does not change the homology class that $S$ represents. If $c$ is primitive, then in particular $c \neq 0$, so that $S \neq \emptyset$.

Suppose that $S_{1}$ and $S_{2}$ are distinct components of $S$. We claim that $M-\left(S_{1} \cup S_{2}\right)$ is not connected. To prove this, we consider disjoint tubular neighborhoods $N_{1}$ and $N_{2}$ of $S_{i}$, and we set $X=M-\operatorname{int}\left(N_{1} \cup N_{2}\right)$. If $M-\left(S_{1} \cup S_{2}\right)$ is connected, so is $X$. Then by Van Kampen's theorem, $\pi_{1}(M)$ is obtained from a free product $\pi_{1}(X) *\left\langle t_{1}\right\rangle *\left\langle t_{2}\right\rangle$, where $\left\langle t_{1}\right\rangle$ and $\left\langle t_{2}\right\rangle$ are infinite cyclic groups, by adding relations of the form $t_{i} \alpha_{i}(x) t_{i}^{-1}=\alpha_{i}^{\prime}(x)$ for each $x \in \pi_{1}\left(S_{i}\right)$ and for $i=1,2$, where $\alpha_{i}$ and $\alpha_{i}^{\prime}$ are suitable homomorphisms from $\pi_{1}\left(S_{i}\right)$ to $\pi_{1}(X)$. It is apparent from this presentation that $\pi_{1}(M)$ admits a homomorphism onto a free group of rank 2 . As this contradicts the hypothesis that $\pi_{1}(M)$ has no free quotient, it follows that $M-\left(S_{1} \cup S_{2}\right)$ is indeed not connected.

We next claim that any two components $S_{1}$ and $S_{2}$ of $S$ represent the same element of $H_{2}(M, \mathbb{Z})$ up to sign. We may assume that $S_{1}$ and $S_{2}$ are distinct. We define $N_{1}, N_{2}$ and $X$ as above. Let $C$ be any component of $X$. Then $\partial C$ is a union 
of components of the 2-manifold $\partial N_{1} \cup \partial N_{2}$, which has four components. Now $\partial C$ cannot be connected, since no component of $S$ separates $M$; and we cannot have $\partial C=\partial N_{1}$, for this would imply that $C \cup N_{1}$ was an open closed subset of $M$ disjoint from $N_{2}$, in contradiction to the connectedness of $M$. Hence $\partial C \not \subset \partial N_{1}$. Similarly $\partial C \not \subset \partial N_{2}$. Hence $\partial C$ must contain at least one component of each $\partial N_{i}$. But we have shown that $X$ is disconnected, and if $C^{\prime}$ is another component of $X$, then $C^{\prime}$ must also contain at least one component of each $\partial N_{i}$. It follows that $\partial C$ consists precisely of one component of $\partial N_{1}$ and one component of $\partial N_{2}$. This immediately implies that $S_{1}$ and $S_{2}$ are homologous up to sign, as asserted.

Thus all the components of $S$ represent the same element of $H_{2}(M ; \mathbb{Z})$ up to sign. More precisely, there is an element $c_{0}$ of $H_{2}(M ; \mathbb{Z})$ such that each $S_{i}$ represents either $c_{0}$ or $-c_{0}$. It follows that $c$ is an integer multiple of $c_{0}$. If we assume that $c$ is primitive, we therefore have $c= \pm c_{0}$. Thus any component of $S$ represents the element $c \in H_{2}(M ; \mathbb{Z})$, after changing its orientation if necessary. Since the components of $S$ are incompressible, this completes the proof of Proposition 2.1.

\section{SeCtion 3. Non-Fibroidal homology ClAsses AND HyPERBolid VOlume}

The bulk of this section is devoted to proving Theorem 3.1. We will then deduce Theorem 3.3 and Corollary 3.4 by combining Theorem 3.1 with the results of the preceding sections.

Theorem 3.1. Let $M$ be a closed hyperbolic 3-manifold. Suppose that there is an infinite set $\mathcal{N}$ of primitive elements of $H_{2}(M ; \mathbb{Z})$ such that every element of $\mathcal{N}$ is represented by some connected, incompressible surface which is not a fibroid. Then the volume of $M$ exceeds 0.83 .

The proof of Theorem 3.1 depends on the following lemma, which is a slight variant of [ACCS, Proposition 10.1]. We refer the reader to [ACCS] for the definition of the maximal embedded tube about a closed geodesic, and of the radius of this tube.

Lemma 3.2. Let $M$ be an orientable hyperbolic 3-manifold. Let $C$ be a non-trivial closed geodesic in $M$, and let $l$ denote its length. Let $\gamma$ denote an element of $\pi_{1}(M)$ whose conjugacy class is represented by $C$. Suppose that $\gamma$ lies in the kernel of some homomorphism of $\pi_{1}(M)$ onto a free abelian group of rank 2 . If $R$ denotes the radius of the maximal embedded tube about $C$, we have

$$
\cosh 2 R \geq \frac{e^{2 l}+2 e^{l}+5}{\left(\cosh \frac{l}{2}\right)\left(e^{l}-1\right)\left(e^{l}+3\right)} .
$$

Proof. The conclusion of this lemma is the same as that of Proposition 10.1 of [ACCS]. In the latter result however, in place of the hypothesis that $\gamma$ lies in the kernel of some homomorphism of $\pi_{1}(M)$ onto a free abelian group of rank 2 , one has the assumption that $\log 3$ is a "strong Margulis number" in the sense of [ACCS]. In that proof, one writes $M=\mathbb{H}^{3} / \Gamma$, where $\Gamma$ is a discrete, torsion-free, co-compact subgroup of $\operatorname{Isom}_{+}\left(\mathbb{H}^{3}\right)$. If one identifies $\pi_{1}(M)$ with $\Gamma$, then $\gamma$ becomes identified with a generator of a maximal cyclic subgroup of $\Gamma$ whose axis in $\mathbb{H}^{3}$ projects to $C$. The assumption that $\log 3$ is a strong Margulis number is then used to guarantee that for any element $\eta$ of $\Gamma$ which does not commute with $\gamma$, and any point $z$ on the axis of $\gamma$, the inequality

$$
\frac{1}{1+e^{\alpha}}+\frac{1}{1+e^{l}} \leq \frac{1}{2}
$$


holds if we set $\alpha=\operatorname{dist}(z, \eta \cdot z)$. Hence we need only show that the latter inequality holds under the assumption that $\gamma$ lies in the kernel of some homomorphism of $\pi_{1}(M)$ onto a free abelian group of rank 2 .

More generally, we shall show that under this assumption, if $z$ is any point of $\mathbb{H}^{3}$ and $\eta$ is any element of $\Gamma$ not commuting with $\gamma$, and if we set $\alpha=\operatorname{dist}(z, \eta \cdot z)$ and $\beta=\operatorname{dist}(z, \gamma \cdot z)$, then

$$
\frac{1}{1+e^{\alpha}}+\frac{1}{1+e^{\beta}} \leq \frac{1}{2}
$$

According to the Main Theorem of [ACCS], this will be the case if $\gamma$ and $\eta$ freely generate a topologically tame subgroup of the purely loxodromic, discrete, torsion-free group $\Gamma \leq \operatorname{Isom}_{+}\left(\mathbb{H}^{3}\right)$. Now suppose that $\gamma$ lies in the kernel of a homomorphism $h$ of $\Gamma$ onto a rank-2 free abelian group $L$. Clearly $L / h(\eta)$ admits a homomorphism $h^{\prime}$ onto $\mathbb{Z}$. Thus $h^{\prime} \circ h$ is a homomorphism of $\Gamma$ onto $\mathbb{Z}$ whose kernel contains $\gamma$ and $\eta$. It then follows from [CS2, Prop. 2.2] and [CS1, Prop. 10.2] that $\gamma$ and $\eta$ generate a topologically tame group, which concludes the proof.

Proof of Theorem 3.1. Since $H_{2}(M ; \mathbb{Z})$ has infinitely many primitive elements, the first Betti number $\beta_{1}(M)$ is at least 2. If $\beta_{1}(M) \geq 3$, then the volume of $M$ exceeds 0.92 according to [CS1, Corollary 10.4]. Thus we may assume that $\beta_{1}(M)=2$. Hence the quotient of $H_{1}(M, \mathbb{Z})$ by its torsion subgroup is a free abelian group $L$ of rank 2 . We let $h: \pi_{1}(M) \rightarrow L$ denote the natural homomorphism.

We set $\lambda=1.0616$, and we distinguish two cases. First consider the case in which $M$ contains a non-trivial closed geodesic $C$ of some length $l<\lambda$ such that the conjugacy class represented by $C$ is contained in the kernel of $h$. Then by Lemma 3.2, the radius $R$ of the maximal embedded tube $T$ about $C$ satisfies

$$
\cosh 2 R \geq \frac{e^{2 l}+2 e^{l}+5}{\left(\cosh \frac{l}{2}\right)\left(e^{l}-1\right)\left(e^{l}+3\right)} .
$$

As is pointed out in [ACCS] at the beginning of Section 10, the volume of $T$ is $\pi l \sinh ^{2} R$. Since $\sinh ^{2} R=\frac{1}{2}(\cosh 2 R-1)$, the volume of $T$ is greater than or equal to $V(l)$, where $V(x)$ is the function defined for $x>0$ by

$$
V(x)=\frac{\pi x}{e^{x}-1}\left(\frac{e^{2 x}+2 e^{x}+5}{2\left(\cosh \frac{x}{2}\right)\left(e^{x}+3\right)}\right)-\frac{\pi x}{2} .
$$

On the other hand, according to [ACCS, Prop. 10.4], the function $V(x)$ decreases monotonically for $x>0$. Since $l<\lambda$ it follows that $V(l)>V(\lambda)=0.8364 \ldots$. Hence in this case 0.83 is a lower bound for the volume of $T$, and therefore also for the volume of $M$.

Now consider the case in which no non-trivial closed geodesic of length $<\lambda$ represents a conjugacy class contained in the kernel of $h$. Since $M$ is closed, there are only a finite number $n \geq 0$ of conjugacy classes in $\pi_{1}(M)$ that are represented by closed geodesics of length $<l$. Let $\gamma_{1}, \ldots, \gamma_{n}$ be elements belonging to these $n$ conjugacy classes. Then $\bar{\gamma}_{i}=h\left(\gamma_{i}\right)$ is a non-trivial element of $L$ for $i=1, \ldots, n$. Since $L$ is a free abelian group of rank 2 , there exists, for each $i \in\{1, \ldots, n\}$, a homomorphism $\phi_{i}$ of $L$ onto $\mathbb{Z}$ such that $\phi_{i}\left(\bar{\gamma}_{i}\right)=0$. Because $\bar{\gamma}_{i} \neq 0$, the epimorphism $\phi_{i}$ is unique up to sign. 
The epimorphism $\phi_{i} \circ h: \pi_{1}(M) \rightarrow \mathbb{Z}$ corresponds to a primitive element of $H^{1}(M ; \mathbb{Z})$, whose Poincaré dual in $H_{2}(M ; \mathbb{Z})$ we shall denote by $c_{i}$. Since the set $\mathcal{N} \subset H_{2}(M ; \mathbb{Z})$ given by the hypothesis of the theorem is infinite, there is an element $c$ of $\mathcal{N}$ which is distinct from $\pm c_{i}$ for $i=1, \ldots, n$. Since $c \in \mathcal{N}$ it follows from the hypothesis that there is a connected incompressible surface $S \subset M$ which represents the homology class $c$ and is not a fibroid.

We now apply Theorem A of [CS3], which asserts that if $S$ is a connected nonfibroid incompressible surface in a closed, orientable hyperbolic 3-manifold $M$, and if $\lambda$ is any positive number, then either (i) $M$ contains a non-trivial closed geodesic of length $<\lambda$ which is homotopic in $M$ to a closed curve in $M-S$, or (ii) $M$ contains a hyperbolic ball of radius $\lambda / 2$. (We repeat that in [CS3], "fibroids" are defined to be connected. However, since the hypothesis of Theorem A of [CS3] refers only to a connected surface $S$, it remains true under the more general definition of a fibroid.) In the present situation, with $\lambda$ chosen as above, we claim that alternative (i) of the conclusion of Theorem A of [CS3] cannot hold.

Indeed, suppose that $C$ is a non-trivial closed geodesic of length $<\lambda$ with the properties stated in (i). Since $C$ has length $<\lambda$, the conjugacy class represented by $C$ contains $\gamma_{i}$ for some $i \in\{1, \ldots, n\}$. Since $C$ is homotopic to a closed curve in $M-S$ it follows that the image of $\gamma_{i}$ in $H_{1}(M ; \mathbb{Z})$ has homological intersection number 0 with $c$. Thus if $\psi: \pi_{1}(M) \rightarrow \mathbb{Z}$ is the homomorphism corresponding to the Poincaré dual of $c$, we have $\psi\left(\gamma_{i}\right)=0$. Now since $L$ is the quotient of $H_{1}(M)$ by its torsion subgroup, $\psi$ factors as $\phi \circ h$, where $\phi$ is some homomorphism from $L$ to $\mathbb{Z}$. Since $c$ is primitive, $\psi$ is surjective, and hence so is $\phi$. But we have $\phi\left(\bar{\gamma}_{i}\right)=\psi\left(\gamma_{i}\right)=0$. In view of the uniqueness that we observed above for $\phi_{i}$, it follows that $\phi= \pm \phi_{i}$, so that $\psi= \pm \phi_{i} \circ h$ and hence $c= \pm c_{i}$. This contradicts our choice of $c$.

Hence (ii) must hold. The lower bound 0.83 for the volume of $M$ now follows from sphere-packing arguments as in [CS1, Corollary 10.4].

It is now easy to deduce the theorem and corollary that were explained in the introduction.

Theorem 3.3. Let $M$ be a closed hyperbolic 3-manifold. Suppose that there is an infinite set $\mathcal{N}$ of primitive elements of $H_{2}(M ; \mathbb{Z})$ such that no element of $\mathcal{N}$ is represented by a connected fibroid. Then the volume of $M$ exceeds 0.83 .

Proof. If $\pi_{1}(M)$ has a non-abelian free quotient, then by Theorem 1.3 the volume of $M$ exceeds 0.92 . Now suppose that $\pi_{1}(M)$ has no non-abelian free quotient. If $\mathcal{N}$ is the set given by the hypothesis of Theorem 3.3, it now follows from Proposition 2.1 that every element of $\mathcal{N}$ is represented by a connected incompressible surface, which by hypothesis cannot be a fibroid. Thus $\mathcal{N}$ has the properties stated in the hypothesis of Theorem 3.1. The latter result therefore implies that the volume of $M$ exceeds 0.83 .

Note that if $M$ is a 3 -manifold whose first Betti number is at least 2, then $H_{2}(M ; \mathbb{Z})$ has infinitely many primitive elements. Hence Theorem 3.3 implies:

Corollary 3.4. Let $M$ be a closed hyperbolic 3-manifold. Suppose that the first Betti number of $M$ is at least 2 , and that $M$ contains no non-separating, connected fibroid. Then the volume of $M$ exceeds 0.83 . 


\section{REFERENCES}

[ACCS] J. W. Anderson, R. Canary, M. Culler, P. B. Shalen, Free kleinian groups and volumes of hyperbolic 3-manifolds, J. Diff. Geom. 43 (1996), 738-782. CMP 97:02

[CHS] M. Culler, S. Hersonsky and P. B. Shalen, The first Betti number of the smallest closed hyperbolic 3-manifold, preprint.

[CS1] M. Culler and P. B. Shalen, Paradoxical decompositions, 2-generator Kleinian groups, and volumes of hyperbolic 3-manifolds, J. Amer. Math. Soc. 5 (1992), 231-288. MR 93a:57017

[CS2] M. Culler and P. B. Shalen, The volume of a hyperbolic 3-manifold with Betti number 2, Proc. Amer. Math. Soc. 120 (1994), 1281-1288. MR 94f:57012

[CS3] M. Culler and P. B. Shalen, Volumes of hyperbolic Haken manifolds, I, Invent. math 118 (1994), 285-329. MR 95g:57023

[H] M. Hall, Jr., Coset representations in free groups, Trans. Amer. Math. Soc. 67 (1949), 421-432. MR 11:322e

[KS] A. Karass and D. Solitar, On finitely generated subgroups of a free group, Proc. Amer. Math. Soc. 22 (1969), 209-213. MR 39:6961

[T] W. P. Thurston, A norm for the homology of 3-manifolds, Memoirs of the Amer. Math. Soc. No. 339, Volume 59, 1986, pp. 99-130. MR 88h:57014

[W] J. Weeks, Hyperbolic structures on three-manifolds, Thesis, Princeton University, 1985.

Department of Mathematics (M/C 249), University of Illinois at Chicago, 851 S. Morgan St., Chicago, Illinois 60607-7045

E-mail address: culler@math.uic.edu

E-mail address: shalen@math.uic.edu 